\title{
Anticoagulation in Very Old Patients with Atrial Fibrillation (AVOPA): A Descriptive Observational Study
}

\author{
Maximilian Hupfer ${ }^{1}$ (D) Markus Gosch ${ }^{1,2}$ (D)
}

Accepted: 14 May 2021 / Published online: 11 June 2021

(c) The Author(s) 2021

\begin{abstract}
Background In older patients with nonvalvular atrial fibrillation, oral anticoagulation is challenging, especially among very old patients. Even though positive effects of oral anticoagulation have been described in this age group ( $>85$ years), there is still a high rate of inappropriate dosing.

Objective This study examines the quality of care for very old hospitalized patients. The aims of this study were to (1) describe the percentage of patients receiving oral anticoagulation at discharge, (2) describe the quality of drug management at discharge, regarding dosing and contraindications, and (3) provide additional data towards establishing a benchmark for the quality of care in very old patients with atrial fibrillation.

Methods This study is a single-center descriptive observational study. The data from 407 patients aged $>85$ years who were hospitalized in 2018 with nonvalvular atrial fibrillation were collected retrospectively from the patient charts. The assessment included specific geriatric aspects, such as falls, the Charlson Comorbidity Index, and nursing categorization.

Results During hospitalization, the proportion of anticoagulated patients increased from $57.5 \%(n=234)$ to $67.3 \%(n=$ $274)$. We found an increasing trend in the use of direct oral anticoagulants, with an increase from $39.8 \%(n=162)$ to $46.2 \%$ $(n=188)$. Regarding the quality of drug management, $13.8 \%(n=56)$ of the patients were not anticoagulated even in the absence of a recognizable contraindication, whereas a contraindication was ignored in only $0.8 \%$ ( $n=2$ out of 188). Dosing was appropriate among all patients taking rivaroxaban, edoxaban, and dabigatran. The dose of apixaban was not reduced in $23.6 \%(n=21$ out of 89$)$, although this dose reduction was necessary according to the dose adjustment criteria. An underdose of a direct oral anticoagulant was found in $26.1 \%$ of the patients ( $n=49$ out of 188).

Conclusions Compared to the results reported in the literature, the percentage of very old patients with atrial fibrillation receiving anticoagulants was high. During hospitalization, the proportion of patients receiving a direct oral anticoagulant increased, which was in contrast to the trend in the proportion of patients taking phenprocoumon. Our results could help to find a benchmark for anticoagulation management among hospitalized very old patients.
\end{abstract}

Maximilian Hupfer

MaxHupfer@googlemail.com

1 Faculty of Medicine, Paracelsus Medical University Nuremberg, General Hospital Nuremberg,

Prof.-Ernst-Nathan-Str. 1, 90419 Nuremberg, Germany

2 Department of Geriatrics, Paracelsus Medical University Nuremberg, General Hospital Nuremberg, Nuremberg, Germany

\section{Key Points}

There is an increasing trend in the administration of direct oral anticoagulants in hospitalized very old patients.

There is potential for improvement in drug management with regard to dosage.

The AVOPA study data could help to find a benchmark for anticoagulation management in the very old patient group. 


\section{Background}

The prevalence of atrial fibrillation (AF) increases with age, reaching $15 \%$ in the group aged older than 85 years [1]. For this reason, oral anticoagulation (OAC) is of particular importance for the prevention of stroke and systemic thromboembolism [2-5]. For a long time, vitamin $\mathrm{K}$ antagonists, such as phenprocoumon and warfarin, were the only available OACs. Only approximately half of the patients received this drug therapy, which shows clear drug undertreatment [6]. Withholding OACs increases the risk of stroke, particularly in very old patients. Currently, direct oral anticoagulants (DOACs) are the first choice for patients with newly diagnosed AF because they have a more favorable risk-benefit profile. For example, intracranial hemorrhage, which is a serious complication of treatment with OACs, occurs significantly less frequently when patients are treated with DOACs than when they are treated with vitamin $\mathrm{K}$ antagonists [7]. Regarding the primary efficacy endpoint, DOACs were at least not inferior to warfarin [8-12].

Therefore, especially in old age, many people benefit from OACs [2]. There are over 2 million patients diagnosed with AF in the USA, and an equally large number of undiagnosed cases is thought to exist. The prevalence of AF is estimated to double by 2050 [3]. In North America, Australia, and Western Europe, approximately $70 \%$ of all patients with AF are over 65 years of age [13, 14].

According to the European Society of Cardiology guidelines for the management of AF, the essential parameters used to adjust the dose are age, body weight, and renal function. In particular, with regard to renal insufficiency, these parameters can ultimately lead to contraindications $[15,16]$. It is important to administer an appropriate dose because a DOAC dose that is too low does not reduce the risk of stroke $[2,17]$. With this in mind, the aim of this study was to determine the quality of care of very old patients with AF in a geriatric department.

Because of the very limited data available on very old patients ( $>85$ years) with $\mathrm{AF}$ and $\mathrm{OAC}$, we wanted to supplement the data for this patient group with our study to be able to guarantee the best possible care. The data from the present study on anticoagulation in very old patients with AF (AVOPA) could help to find a benchmark for comparing the quality of OACs in a very old group patients with comorbities. First, the percentage of very old, hospitalized patients with AF who received OACs was calculated. Second, the drug that was most commonly prescribed by geritricians was determined. The quality of the medication was then examined and the proportions of patients who received the appropriate dose and whom the contraindications were correctly taken into account were calculated. Finally, we assessed the extent to which our data with regard to the parameters of the $\mathrm{CHADS}_{2}$ score were comparable to data from randomized controlled trials (RCTs) of DOACs.

\section{Patients and Methods}

\subsection{Study Design and Population}

In the present, single-center descriptive observational study, all patients aged $>85$ years with nonvalvular AF, who were admitted to a geriatric department in 2018, were included. A very old patient group was generated. Patients who died during hospitalization were excluded to create a common starting and ending point and thus the opportunity to investigate changes in OACs during hospitalization. Only the data from hospitalized patients were collected because the aim was to investigate hospital care. Outpatients were excluded. In total, the data from 407 patients were collected retrospectively. The information was collected from medical histories and patient charts. Because of the comprehensive documentation and the standardized process, there were no missing data for the parameters collected. The data were anonymized and evaluated. All patient characteristics can be seen in the Electronic Supplementary Material.

Weight, height, body mass index, systolic blood pressure, diastolic blood pressure, urea, serum creatinine, estimated glomerular filtration rate, and activities of daily living were measured immediately before discharge. Because of the lack of the measurement of creatinine clearance, the estimated glomerular filtration rate was used as a substitute in this study. The need for nursing care was assessed by using a score from 1 (independent) to 4 (completely dependent). The level of nursing care included the following service areas: self-care, nutrition, toileting, motor skills, safety, and communication. In grade 1 , no nursing assistance is necessary. In grade 2 , help is required with the preparation and follow-up of activities, but the patient can mostly be left alone. In grade 3 , it is necessary for the staff to take charge of the activities, and the nurse must always be present when the patient is performing the activities. In grade 4, full support is required during the activity.

Furthermore, the Barthel Index was used, which measures the likelihood of being able to live at home with a degree of independence following discharge from hospital [18]. Modified by Granger, Dewis, Peters, Sherwood, and Barrett and refined by Shah, Vanclay, and Cooper, the index includes ten basic activities of daily living: bowels, bladder, grooming, toilet use, feeding, transfers, walking, dressing, climbing stairs, and bathing. Every item is scored $0-10$ points, with a total score of $0-100[19,20]$. 
In addition, the $\mathrm{CHADS}_{2}$ score, the $\mathrm{CHA}_{2} \mathrm{DS}_{2}$-VASc score, and the HAS-BLED score were calculated based on information obtained from the patient charts [21-23]. The $\mathrm{CHADS}_{2}$ score and the $\mathrm{CHA}_{2} \mathrm{DS}_{2}$-VASc score indicate the clinical risk of stroke, and the HAS-BLED score shows the clinical risk of bleeding.

The Charlson Comorbidity Index (CCI) was used to measure the burden of disease. The index was developed to measure 1-year mortality based on 19 different comorbidities. Higher scores indicate higher burdens of comorbidities with a maximum of $85 \%$ with $>5$ points [24]. The process of camparing the groups is shown in the flowchart (Fig. 1).

First, we compared patient characteristics between the group taking OACs and those not taking anticoagulants. Second, we analyzed the group of patients receiving an inappropriately low dose of a DOAC and checked all patients for contraindications. Third, we compared the data from our study population with the data from the DOAC RCTs using the $\mathrm{CHADS}_{2}$ score.

\subsection{Statistics}

The data analysis was carried out with SPSS Statistics 22, Version 22.0.0.0 (IBM, Ehningen, Baden-Württemberg, Germany). Descriptive data are shown as numbers, percentages, mean values, and standard deviations.

The Mann-Whitney $U$ test for independent samples was selected to check the significance of the differences in mean values for continuous variables. Furthermore, we calculated the median and the interquartile range to enable a better comparison. A $p$ value $<0.05$ indicated significance, and $95 \%$ confidence intervals were calculated. Chi-square tests were used to determine significance.

\subsection{Ethics Consideration}

This was a retrospective study with anonymized data, and approval was given by the institutional review board of the hospital.

\section{Results}

\subsection{Main Outcome}

During hospitalization, the proportion of patients receiving OACs increased from 55.3 to $59.4 \%$. In total, $67.3 \%$ of the patients were receiving anticoagulants at hospital discharge. We found that the proportion of patients receiving DOACs increased from 39.8 to $46.2 \%$, while the proportion of patients treated with phenprocoumon decreased from 15.5 to $13.2 \%$.

\subsection{Secondary Outcomes}

Apixaban was the most common drug used for anticoagulation $(26.5 \%)$. A total of $13.8 \%$ of the study population with missing or unrecognizable contraindications did not receive any anticoagulation. We compared this group with the $59.4 \%$ of patients receiving OACs. The Barthel Index and the $\mathrm{CHA}_{2} \mathrm{DS}_{2}$-VASc score were higher in the group receiving OACs.

A reduced dose was prescribed to $31.0 \%$ of the patients ( $n=49$ out of 158 patients with a reduced DOAC dose), although the criteria for a reduced dose were not met. This patient group was underdosed. A comparison of this group of patients with the group with the correct dosage showed that the former patients were on average older, had higher serum creatinine levels, were significantly more likely to
Fig. 1 Flowchart. $A F$ atrial fibrillation, $A V O P A$ anticoagulation in very old patients with atrial fibrillation, $D O A C$ direct oral anticoagulant, $O A C$ oral anticoagulation

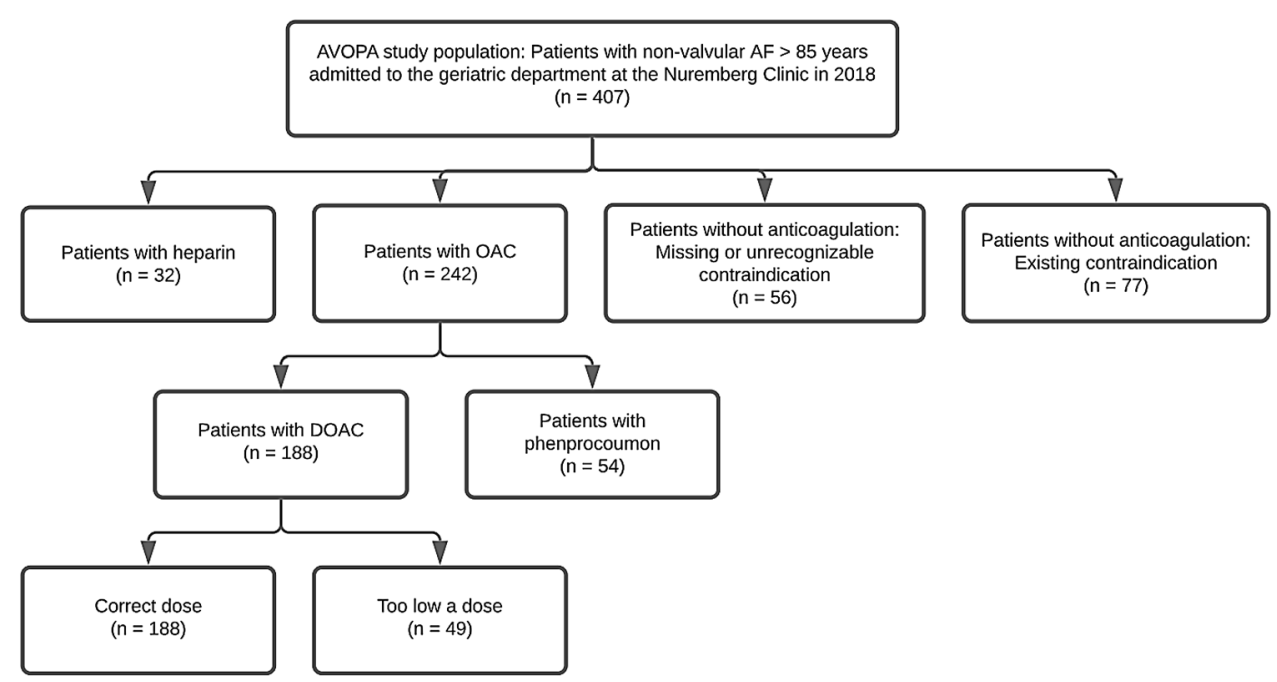


have fallen in the last 8 weeks, and had a higher CCI score. A further comparison of our data with the data from the DOAC RCT showed similar values for the $\mathrm{CHADS}_{2}$ score in both populations.

\subsection{Type of Anticoagulation}

At the time of hospital admission, $57.5 \%(n=234)$ of the patients were already receiving an anticoagulant. A total of $55.3 \%$ ( $n=225)$ were taking an OAC at hospital admission. By discharge, the proportion of patients taking an anticoagulant had increased to $67.3 \%(n=274)$, and the proportion of patients taking OACs had risen to $59.4 \%(n=242)$. There was a relative increase of $17.1 \%(n=40$ out of 234$)$ in patients receiving anticoagulants and a relative increase of $7.6 \%$ ( $n=17$ out of 225$)$ in patients taking OACs (Table 1$)$.

In $46.2 \%$ ( $n=188)$ of the cases, a DOAC was prescribed. Apixaban was the most commonly prescribed drug in $26.5 \%$ of the patients $(n=108)$, followed by rivaroxaban in $10.6 \%$ $(n=43)$ and edoxaban in $7.4 \%(n=30)$. Dabigatran was only used in $1.7 \%$ of the patients $(n=7)$. Phenprocoumon was the next most common drug after DOACS and was prescribed to $13.2 \%$ of the patients $(n=54)$. Heparin and lowmolecular-weight heparin were used in $7.9 \%$ of the patients $(n=32)$, usually in connection with bridging, during interventions or in the case of severe renal failure (Figs. 2, 3). The patients who were discharged with heparin were recommended to switch to another drug as an outpatient. Patients were also transferred to other departments on heparin. Detailed data were not collected. However, this group was by definition excluded from the group of patients with OACs to avoid affecting the results.

During hospitalization, we found an increase in the proportion of patients on DOACs from $39.8 \%(n=162)$ to $46.2 \%(n=188)$, which was a relative increase of $16.0 \%$ $(n=26)$. Accordingly, we observed a relative decrease of $14.3 \%(n=9)$ in the proportion of patients treated with phenprocoumon, which decreased from $15.5 \%(n=63)$ to $13.2 \%(n=54)$. The most clinically relevant change, which was a relative increase of $36.7 \%(n=29)$, was found in the group of patients taking apixaban. The proportion rose

Table 1 Type of anticoagulant

Anticoagulant treatment at hospital admission ${ }^{\mathrm{a}}$, no./total (\%)

$234 / 407(57.5)$

Anticoagulant treatment at discharge ${ }^{\mathrm{a}}$, no./total (\%)

$274 / 407(67.3)$

No anticoagulant treatment at discharge, no./currently not taking an anticoagulant (\%)

$133 / 407(32.7)$

Existing contraindication, no./currently not taking an anticoagulant (\%)

$77 / 133(57.9)$

Missing or unrecognizable contraindication, no./currently not taking an anticoagulant (\%)

$56 / 133(42.1)$

$\mathrm{OAC}$ at hospital admission, no./total (\%)

$225 / 407(55.3)$

DOAC, no./total (\%)

$162 / 407$ (39.8)

Phenprocoumon, no./total (\%)

$63 / 407(15.5)$

Other substances at hospital admission

Antiplatelet agent, no./total (\%)

$50 / 407$ (12.3)

Heparin, no./total (\%)

9/407 (2.2)

OAC at discharge, no./total (\%)

242/407 (59.4)

DOAC, no./total (\%)

$188 / 407$ (46.2)

Phenprocoumon, no./total (\%)

$54 / 407$ (13.2)

Other substances at discharge

Antiplatelet agent, no./total (\%)

$52 / 407$ (12.7)

Heparin, no./total (\%)

$32 / 407(7.9)$

DOAC at discharge

Apixaban, no./total (\%)

$108 / 407(26.5)$

Reduced dose, no./apixaban (\%)

$85 / 108(78.7)$

Rivaroxaban, no./total (\%)

43/407 (10.6)

Reduced dose, no./rivaroxaban (\%)

$40 / 43(93.0)$

Edoxaban, no./total (\%)

$30 / 407(7.4)$

Reduced dose, no./edoxaban (\%)

$26 / 30(86.7)$

Dabigatran, no./total (\%)

$7 / 407(1.7)$

Reduced dose, no./dabigatran (\%)

$7 / 7(100)$

$D O A C$ direct oral anticoagulant, $O A C$ oral anticoagulant

${ }^{a}$ Including DOACs, phenprocoumon and heparin 
Fig. 2 Anticoagulation therapy at discharge
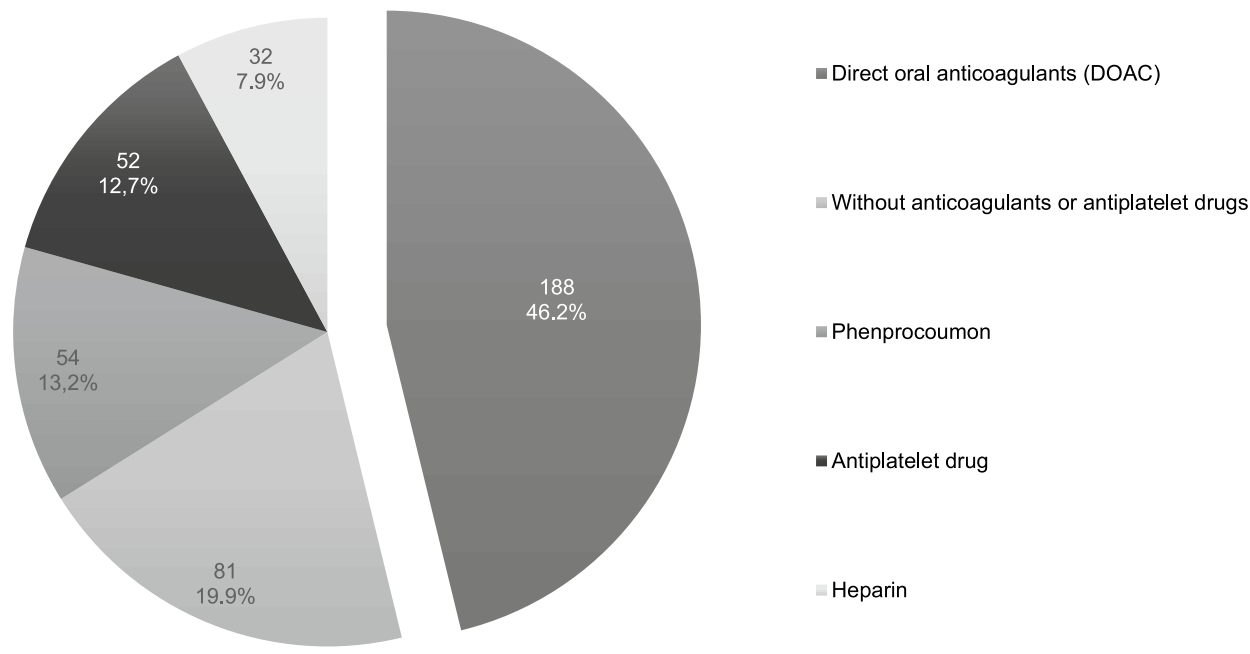

Fig. 3 Direct oral anticoagulants (DOACs) at discharge

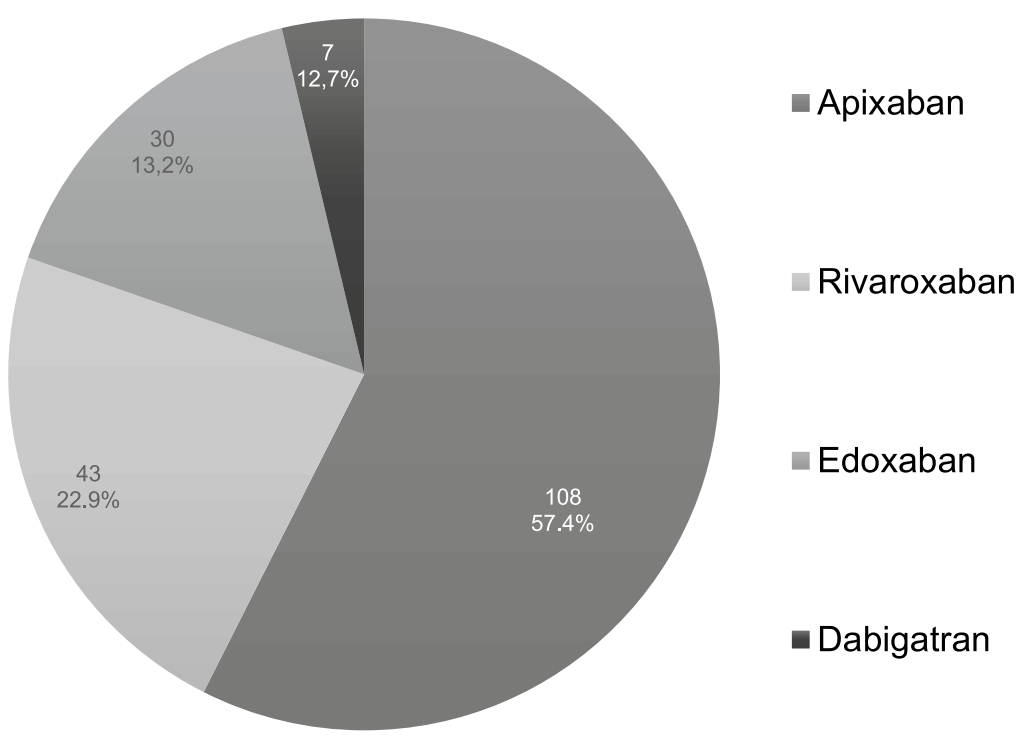

from $19.4 \%(n=79)$ to $26.5 \%(n=108)$. Figure 4 shows the changes in absolute numbers.

\subsection{Comparison of Patients with OACs and without Anticoagulants}

Only $0.8 \%$ of the patients ( $n=2$ out of 188 ) were receiving an OAC despite a contraindication. In total, $32.7 \%$ ( $n$ $=133$ ) of the patients were not receiving an oral anticoagulant or heparin. In this group of 133 patients who were not undergoing anticoagulation at discharge, a contraindication was found in $57.9 \%(n=77$ out of 133). Accordingly, $42.1 \%$ ( $n=56$ out of 133 ) had missing or unrecognizable contraindications. This accounted for $13.8 \%$ $(n=56)$ of the total study population of 407 patients. To identify the reasons for withholding anticoagulation from these 56 patients with missing or unrecognizable contraindications, these patients were compared with the group of 242 patients receiving OACs (Table 2).

The Barthel Index differed between the two groups by 19.4 points, with the higher value of 57.4 points in the group taking OACs and the lower value of 38.0 points in the group not undergoing anticoagulation. The difference was statistically significant, with a $p$ value of $<0.001$. The $\mathrm{CHA}_{2} \mathrm{DS}_{2}$-VASc score of 4.4 points in the group not undergoing anticoagulation was 0.4 points lower than the 4.8 points in the group taking OACs. The difference was not statistically significant, with a $p$ value of 0.114 .

\subsection{Comparison of Patients with Correct DOAC Doses and Inappropriately Low Doses}

With regard to the recommended dose adjustment criteria, the dose was reduced in $84.0 \%$ ( $n=158$ out of 188 ) of the 


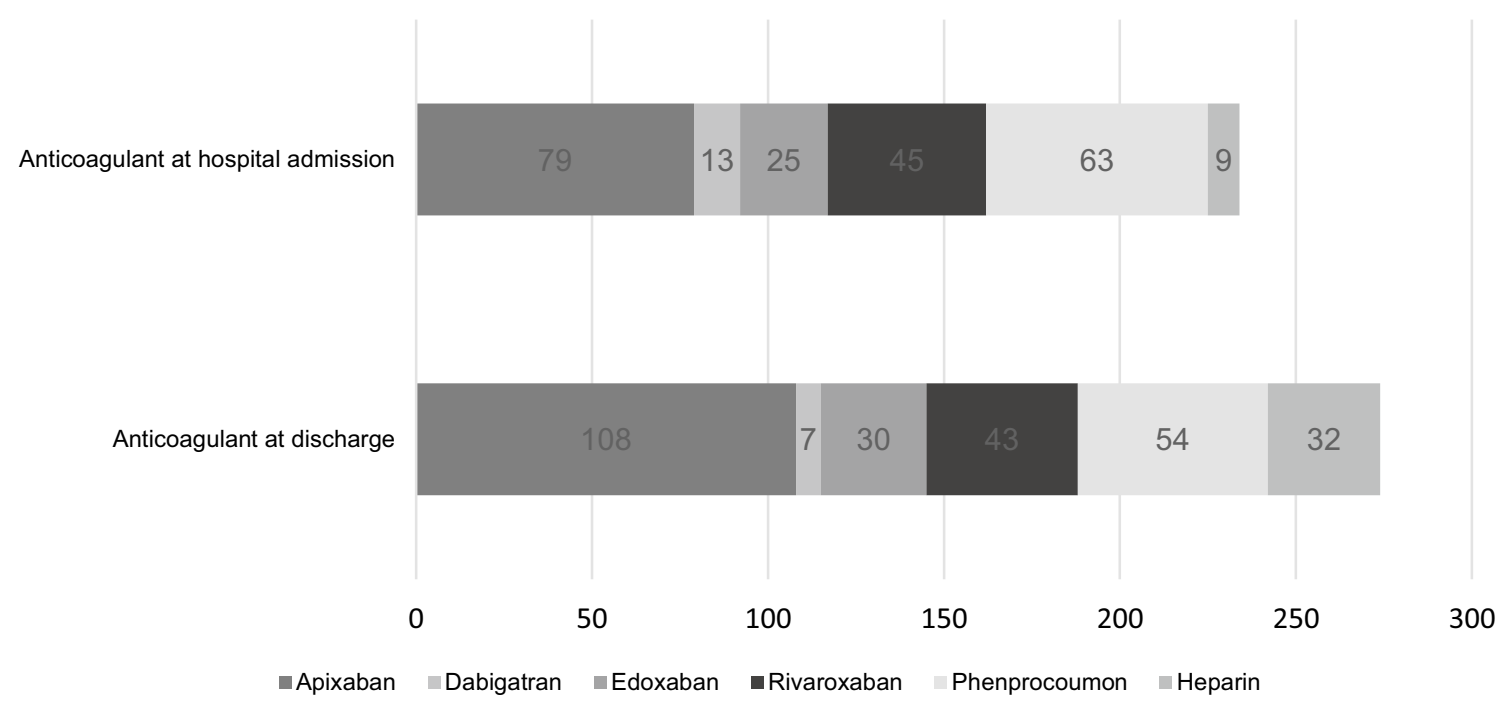

Fig. 4 Substance at hospital admission and anticoagulant at discharge

Table 2 Differences between the patient groups: patients with OACs and those not undergoing anticoagulation with missing or unrecognizable contraindications

\begin{tabular}{|c|c|c|c|c|c|c|c|}
\hline & \multicolumn{3}{|l|}{ With OAC } & \multicolumn{3}{|c|}{ No anticoagulation $^{\mathrm{a}}$} & \multirow[t]{2}{*}{$p$ value } \\
\hline & & Median & IR & & Median & IR & \\
\hline Age, years & $90.3 \pm 3.1$ & 90.0 & 4.0 & $91.0 \pm 3.2$ & 91.0 & 6.0 & 0.115 \\
\hline Weight $^{\mathrm{b}}, \mathrm{kg}$ & $67.5 \pm 14.2$ & 65.8 & 19.3 & $72.6 \pm 10.7$ & 71.1 & 13.3 & 0.026 \\
\hline Number of drugs ${ }^{\mathrm{b}}$, no. & $9.0 \pm 3.4$ & 9.0 & 4.0 & $7.2 \pm 2.8$ & 7.0 & 4.0 & 0.001 \\
\hline Serum creatinine $^{\mathrm{b}}, \mathrm{mg} / \mathrm{dL}$ & $1.2 \pm 0.4$ & 1.1 & 0.5 & $1.0 \pm 0.3$ & 1.0 & 0.5 & 0.006 \\
\hline eGFR $>60^{\mathrm{b}}$, no./total $(\%)$ & $80 / 242(33.1)$ & - & - & $25 / 56(44.6)$ & - & - & 0.102 \\
\hline $\mathrm{eGFR} \leq 60^{\mathrm{b}}, \mathrm{mL} / \mathrm{min}$ & $42.8 \pm 10.6$ & 43.0 & 17.0 & $48.2 \pm 9.5$ & 52.0 & 16.0 & 0.011 \\
\hline Barthel Index on the day of discharge, points & $57.4 \pm 26.3$ & 60.0 & 30.0 & $38.0 \pm 29.4$ & 35.0 & 55.0 & $<0.001$ \\
\hline Level of basic care ${ }^{\mathrm{b}}$, level & $2.4 \pm 0.8$ & 2.0 & 1.0 & $3.1 \pm 1.0$ & 3.0 & 1.8 & $<0.001$ \\
\hline $\mathrm{CHA}_{2} \mathrm{DS}_{2}$ VASc score, points & $4.8 \pm 1.5$ & 5.0 & 2.0 & $4.4 \pm 1.5$ & 4.0 & 3.0 & 0.114 \\
\hline HAS-BLED score, points & $2.1 \pm 0.7$ & 2.0 & 1.0 & $2.2 \pm 0.6$ & 2.0 & 1.0 & 0.476 \\
\hline Charlson Comorbidity Index, points & $2.7 \pm 1.8$ & 3.0 & 3.0 & $2.5 \pm 2.0$ & 2.0 & 2.0 & 0.208 \\
\hline
\end{tabular}

$\mathrm{CHA}_{2} \mathrm{DS}_{2}$-VASc score: clinical risk factor for stroke, transient ischemic attack, and systemic embolism; higher scores indicate a greater risk; congestive heart failure, hypertension, diabetes mellitus, vascular disease, an age from 65 to 74 years and female sex (1 point each), an age of 75 years or older, prior stroke or transient ischemic attack (2 points each)

HAS-BLED score: clinical risk factor for bleeding; higher scores indicate a greater risk; hypertension, abnormal renal/liver function, stroke, bleeding history or predisposition, labile INR, elderly status (age $>65$ years), drugs/alcohol concomitantly ( 1 point each)

Charlson Comorbidity Index; 1-year risk of mortality for a patient who may have a range of comorbid conditions; higher scores indicate a higher risk of mortality

Level of basic care: the need for basic care was assessed by using a score from 1 (independent) to 4 (completely dependent). The level of nursing care included the following service areas: self-care, nutrition, toileting, motor skills, safety, and communication

Plus-minus values are means \pm standard deviation; determination of the $p$ value for nominal variables was made with the chi-square test and for continuous variables with the Mann-Whitney $U$ test

$e G F R$ estimated glomerular filtration rate, $I R$ interquartile range, $O A C$ oral anticoagulation

${ }^{\mathrm{a}}$ No anticoagulation with missing or unrecognizable contraindication

${ }^{\mathrm{b}}$ Last measured value 
DOAC group. Apixaban was used in $78.7 \%$ ( $n=85$ out of 108 ) of the patients, and rivaroxaban was used in $93.0 \%$ ( $n=40$ out of 43 ) of the patients. Considering the dose adjustment criteria for rivaroxaban, apixaban, edoxaban, and dabigatran, the required adjustments were made in $100 \%$ ( $n$ $=40$ out of 40$), 76.5 \%$ ( $n=65$ out of 85$), 100 \%$ ( $n=26$ out of 26 ), and $100 \%$ ( $n=7$ out of 7 ) of the patients taking those drugs, respectively.

A reduced dose was prescribed to $31.0 \%$ of the patients ( $n=49$ out of 158), although the adjustment criteria for a reduced dose were not met. A comparison of this group of patients (inappropriately low DOAC dose) with the group of patients with a correct DOAC dose showed that the former patients were on average 1.1 years older $(p=0.045)$. Patients with the correct DOAC doses were 90.1 years old on average, and patients with too low a dose were 91.2 years old on average. The group with too low a dose had a serum creatinine level that was $0.2 \mathrm{mg} / \mathrm{dL}$ higher than that in the group with correct doses $(p=0.009)$. Patients with a correct DOAC dose had, on average, a serum creatinine level of $1.1 \mathrm{mg} / \mathrm{dL}$, and patients with too low a dose had a value of $1.3 \mathrm{mg} / \mathrm{dL}$. In addition, $59.2 \%(n=$
29 out of 49) of the patients who received a dose that was too low had fallen in the past 8 weeks, which was a higher proportion than in the comparison group with the correct doses $(41.7 \% ; n=58$ out of 139$)$. The difference was significant $(p=0.035)$. The CCI score was 3.2 points in the group with inappropriately low doses, which was 0.6 points higher $(p=0.034)$ than in the group with correct doses. The patients with correct doses had, on average, a CCI score of 2.6 points. The difference was significant $(p=0.034)$. No significant differences were seen in the $\mathrm{CHA}_{2} \mathrm{D}_{\mathrm{S}}$-VASc and the HAS-BLED scores (Table 3 ).

\subsection{Comparison of the AVOPA Study with the Randomized Controlled Trials of DOACs}

We compared our study population with those of the DOAC RCTs using the $\mathrm{CHADS}_{2}$-Score (Table 4). The score for patients with warfarin or phenprocoumon was 2.4 in the AVOPA study and 2.1 in the ARISTOLE study, and similar scores were obtained by the patients with normal doses of DOACs in this study and those in ENGAGE
Table 3 Differences between the patient groups with DOACs: correct dose or inappropriately low dose

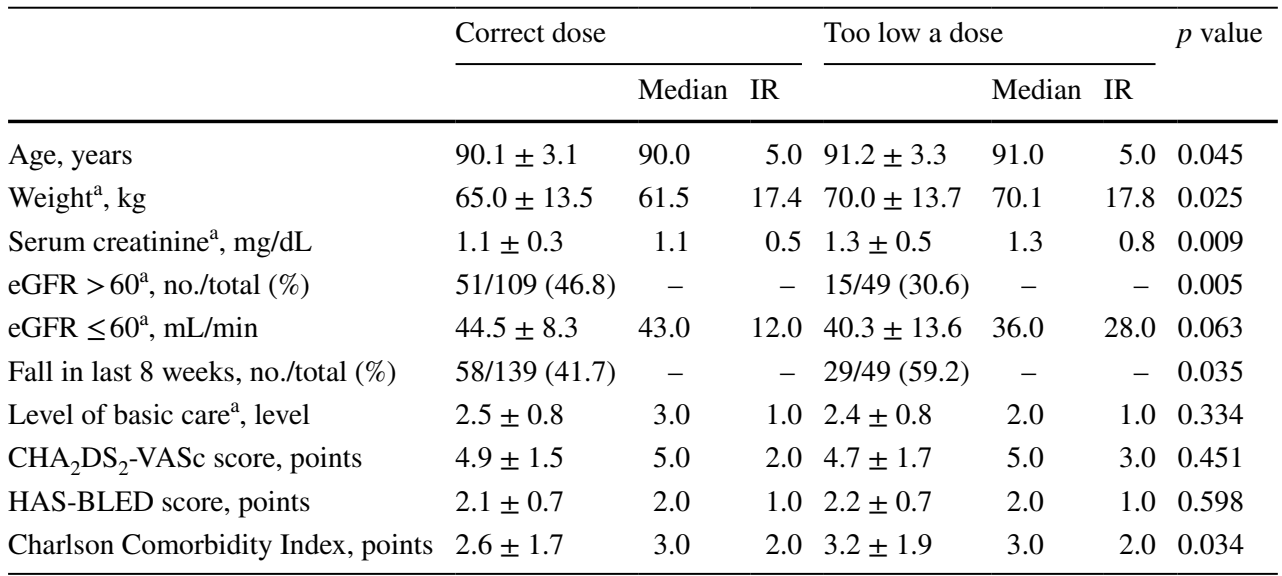

Plus-minus values are means \pm standard deviation; determination of the $p$ value for nominal variables was made using the chi-square test and for continuous variables using the Mann-Whitney $\mathrm{U}$ test

$\mathrm{CHA}_{2} \mathrm{DS}_{2}$-VASc Score: clinical risk factor for stroke, transient ischemic attack and systemic embolism; higher scores indicate a greater risk; congestive heart failure, hypertension, diabetes mellitus, vascular disease, an age from 65 to 74 years and female sex (1 point each), an age of 75 years or older, prior stroke or transient ischemic attack ( 2 points each)

HAS-BLED score: clinical risk factor for bleeding; higher scores indicate a greater risk; hypertension, abnormal renal/liver function, stroke, bleeding history or predisposition, labile INR, elderly status (age $>65$ years), drugs/alcohol concomitantly (1 point each)

Charlson Comorbidity Index: 1-year risk of mortality for a patient who may have a range of comorbid conditions; higher scores indicate a higher risk of mortality

Level of basic care: the need for basic care was assessed by using a score from 1 (independent) to 4 (completely dependent). The level of nursing care included the following service areas: self-care, nutrition, toileting, motor skills, safety, and communication

$e G F R$ estimated glomerular filtration rate, $I R$ interquartile range, $O A C$ oral anticoagulation

${ }^{\text {a }}$ Last measured value 
Table 4 Comparison of the AVOPA study population with the patient groups in the DOAC RCTs based on the CHADS score

\begin{tabular}{lclccc}
\hline $\begin{array}{l}\text { CHADS }_{2} \text { score of the } \\
\text { study population, points }\end{array}$ & All patients & $\begin{array}{l}\text { Patients taking warfa- } \\
\text { rin or phenprocoumon }\end{array}$ & DOAC & $\begin{array}{l}\text { Normal dose } \\
\text { of DOAC }\end{array}$ & $\begin{array}{l}\text { Reduced } \\
\text { dose of } \\
\text { DOAC }\end{array}$ \\
\hline AVOPA & $2.4 \pm 1.1$ & $2.4 \pm 1.1$ & $2.5 \pm 1.1$ & $2.6 \pm 1.1$ & $2.5 \pm 1.2$ \\
RE-LY & - & $2.1 \pm 1.1$ & - & $2.2 \pm 1.2$ & $2.1 \pm 1.1$ \\
ROCKET-AF & - & $3.5 \pm 0.9$ & $3.5 \pm 0.9$ & - & - \\
ARISTOLE & - & $2.1 \pm 1.1$ & $2.1 \pm 1.1$ & - & - \\
ENGAGE AF-TIMI 48 & - & $2.8 \pm 1.0$ & - & $2.8 \pm 1.0$ & $2.8 \pm 1.0$ \\
\hline
\end{tabular}

Plus-minus values are means \pm standard deviation

AVOPA Anticoagulation in Very Old Patients with Atrial Fibrillation: this study, DOAC direct oral anticoagulant, $R C T$ s randomized controlled trials

$\mathrm{CHADS}_{2}$ score: clinical risk factor for stroke; higher scores indicate a greater risk; congestive heart failure, hypertension, an age over 75 years, diabetes mellitus (1 point each), prior stroke or transient ischemic attack ( 2 points each)

DOAC RCTs: RE-LY = Randomized Evaluation of Long-term anticoagulation therapY; dabigatran; ROCKET-AF $=$ Rivaroxaban Once daily oral direct factor Xa inhibition Compared with vitamin K antagonism for prevention of stroke and Embolism Trial in Atrial Fibrillation; rivaroxaban; ARISTOLE = Apixaban für Reduction In Stroke and other ThromboembOlic Events in atrial fibrillation; apixaban; ENGAGE AF-TIMI 48 = Effective ANticoaGulation with Factor XAnext Generation in Atrial Fibrillation-Thrombolysiis in Myocardial Infarction 48; edoxaban
AF-TIMI 48. The patients in this AVOPA study scored 2.6 and those in ENGAGE AF-TIMI 48 scored 2.8.

\section{Discussion}

Because of very limited data available on very old patients (aged $>85$ years) with AF and OAC, we wanted to supplement the data for this patient group with our study. The result should be an improvement in the supply of this patient group to be able to guarantee the best possible care. The data from the AVOPA study could help to find a benchmark for comparing the quality of OACs in very old patients. At the time of hospital discharge, $67.3 \%$ of the patients were receiving anticoagulants. A total of $46.2 \%$ of those were treated with DOACs. According to the dose adjustment criteria, the dose was reduced in $84.0 \%$ of the patients taking DOACs $(n=158$ out of 188). The dose was adjusted in $31.0 \%$ of those patients ( $n=49$ out of 158 ) despite the fact that those patients did not meet the criteria for a reduction in their dose. This indicated that those patients were underdosed. This high proportion of very old patients represents a particular problem with regard to contraindications and, in particular, the fear of bleeding.

Our data are comparable to those of Kirchhof et al. [25]. In their results, they showed a proportion of $68.8 \%$ patients receiving anticoagulants in the setting of a maximum care hospital. In a further comparison of our data with those in the study by Barnes et al. [26] in 2014, the latter reported that $66.9 \%$ of the patients were receiving anticoagulants. In our study, the proportion was higher (67.3\%) [26] (Table 5).
Regarding the proportion of patients receiving anticoagulants, our value was in the upper portion of the range, even though we included only patients aged $>85$ years. In particular, the $17.1 \%$ relative increase in the proportion of patients receiving DOACs and the $14.3 \%$ relative decrease in the proportion of patients treated with phenprocoumon during hospitalization shows that the slightly better risk-benefit profile of DOACs was being taken into account [8-11].

The inclusion criteria of the AVOPA study included the diagnosis of AF. Furthermore, the $\mathrm{CHA}_{2} \mathrm{DS}_{2}$-VASc score was calculated to verify the indication for anticoagulation. In addition, the correct assessment of contraindications was determined. Contraindications were ignored in only 2 out of 407 patients $(0.05 \%)$. For these reasons, the data collected are highly accurate and could serve as a benchmark for the quality of treatment with OACs in a very old (aged $>85$ years) population in routine clinical practice.

The $\mathrm{CHADS}_{2}$ score was similar between this study and the RCTs of DOACs. Therefore, our results can be used to supplement the data available for very old patients [8-11].

A comparison of the data between the current study and that by Barnes et al. [26] showed that although there were similar total numbers of patients with AF and undergoing anticoagulation, there were differences in the treatments selected. For example, in Barnes et al. [26], approximately half of the patients were treated with warfarin, and the other half were treated with a DOAC. In the AVOPA study, however, a DOAC was administered to $68.6 \%$ of the patients undergoing anticoagulation. This reflects the recommendations of the European Society of Cardiology regarding AF from 2016 [16]. The European Society of Cardiology recommends that OACs should be considered for patients with AF 
Table 5 Comparison of different patient cohorts

\begin{tabular}{|c|c|c|c|c|c|c|}
\hline & Age, years & $\begin{array}{l}\text { Antico- } \\
\text { agulation, } \\
\text { no./total } \\
(\%)\end{array}$ & $\begin{array}{l}\text { DOAC, no./DOAC } \\
(\%)\end{array}$ & $\begin{array}{l}\text { DOAC (reduced } \\
\text { dose), no./DOAC } \\
(\%)\end{array}$ & $\begin{array}{l}\text { DOAC (inappro- } \\
\text { priately low dose), } \\
\text { no./DOAC reduced } \\
\text { dose }(\%)\end{array}$ & $\begin{array}{l}\text { Without anticoagula- } \\
\text { tion (with existing } \\
\text { contraindication), no./ } \\
\text { without anticoagula- } \\
\text { tion (\%) }\end{array}$ \\
\hline AVOPA & $90.6 \pm 3.3$ & 67.3 & $\begin{array}{l}\text { Apixaban } 57.4 \\
\text { Rivaroxaban } 22.9 \\
\text { Edoxaban } 13.0 \\
\text { Dabigatran } 3.7\end{array}$ & 84.0 & 31.0 & 57.9 \\
\hline Kirchhof et al. [25] & $68.4 \pm 11.0$ & $\begin{array}{l}68.8 \\
\text { (clinic } \\
\text { of maxi- } \\
\text { mum } \\
\text { care) }\end{array}$ & - & - & - & - \\
\hline Barnes et al. [26] & - & 66.9 & $\begin{array}{l}\text { Apixaban } 26.4 \\
\text { Rivaroxaban } 48.2 \\
\text { Dabigatran: } 25.4\end{array}$ & - & - & - \\
\hline Lee et al. [2] & $66.8 \pm 11.7$ & 100 & - & 41.6 & 20.3 & - \\
\hline Ekerstad et al. [27] & $\begin{array}{l}86.1 \pm 5.1 \text { (patients } \\
\text { with } \mathrm{AF} \text { ) }\end{array}$ & 62.6 & $\begin{array}{l}\text { Apixaban } 84.2 \\
\text { Rivaroxaban } 10.5 \\
\text { Dabigatran } 5.3\end{array}$ & - & - & 56.3 \\
\hline Lefebvre et al. [28] & $\begin{array}{l}87.4 \pm 4.96 \text { (no } \\
\text { anticoagulation) } \\
85.3 \pm 3.94 \text { (antico- } \\
\text { agulation) }\end{array}$ & 70.0 & - & - & - & - \\
\hline
\end{tabular}

Plus-minus values are means \pm standard deviation

$D O A C$ direct oral anticoagulant

and a $\mathrm{CHA}_{2} \mathrm{DS}_{2}$-VASc score of 1 and are indicated in those with a score of 2 or more points. Because of the slightly better risk-benefit profile, DOACs should be preferentially selected [8-11]. In the study by Barnes et al. [26], rivaroxaban was the most common DOAC, and it accounted for $48.2 \%$, followed by apixaban, which accounted for $26.4 \%$. In the AVOPA study, however, apixaban accounted for $57.4 \%$ of the DOACs, and rivaroxaban accounted for $22.9 \%$. The reason for the high proportion of patients who were treated with apixaban may be the consistent use of the OAC-FORTA 2016 guidelines at the Nuremberg Clinic. The FORTA classification system is a means of assessing the suitability of drugs in patients of different ages. Apixaban was convincingly ranked first, with evidence in old patients, and was the only substance in the best category, A. The remaining DOACs were assigned to the second group, category B [29]. This also included the vitamin $\mathrm{K}$ antagonist warfarin. The application of the FORTA system showed a clearly positive effect in an intervention study [30].

In the AVOPA study, $84.0 \%$ ( $n=158$ out of 188$)$ of patients treated with a DOAC took a reduced dose. When comparing the data with those from Lee et al. [2], who examined the "Effectiveness and Safety of Off-label Dosing of Nonvitamin K Antagonist Anticoagulant for Atrial Fibrillation in Asian Patients" and reported that $41.6 \%$ of the patients took a reduced dose, the proportion in the current study was significantly higher. The reason for this significant difference is the very old population in the AVOPA study and the numerous comorbidities associated with old age. For example, the mean age of patients in the AVOPA study was 90.6 years, compared to 66.8 years in the study by Lee et al. [2]. With regard to the dose adjustment criteria, it was found that in $31.0 \%$ ( $n=49$ out of 158) of the patients taking a reduced dose, the criteria for a dose reduction were not met. These patients received a dose that was inappropriately low. A comparison of our data with those from Lee et al. [2] showed very similar values. Lee et al. [2] reported a value of $20.3 \%$.

In addition, it is clear that in $23.6 \%$ ( $n=21$ out of 89 ) of patients, the dose of apixaban was not reduced, although a reduction was indicated by the adjustment criteria. These patients received an inappropriately high dose. A possible reason for this is the way data were collected for the AVOPA study. For example, for the serum creatinine and creatinine clearance values, only the last measured value was taken into account. Only a point value was used, and fluctuations in and the trajectories of these parameters were not considered.

Low creatinine clearance and high serum creatinine levels are fixed contraindication criteria for treatment with DOACs. Therefore, $32.7 \%(n=133)$ of the patients in the AVOPA study were not receiving anticoagulants at hospital discharge. Of this group, $57.9 \%(n=77$ out of 133$)$ had 
an existing contraindication, such as a creatinine clearance value lower than $30 \mathrm{~mL} / \mathrm{min}$. We compared these data with the results of Ekerstad et al. [27]. This is comparable to the $56.3 \%$ reported by Ekerstad et al. [27]. The comparison of the two studies is useful because the patient populations were similar in several essential aspects. Ekerstad et al.'s study also had a very old patient population aged $\geq 75$ years. That study included 190 patients with AF. Similar to the AVOPA study, Ekerstad et al. [27] compared patients with $\mathrm{AF}$ who were and were not undergoing anticoagulation. One slight difference was the inclusion of patients with heparin in the anticoagulated group in the study by Ekerstad et al. [27]. In the present study, in contrast, a comparison was made with patients treated with OACs, and heparinized patients were excluded from this group. However, the percentage of heparinized patients in the study by Ekerstad et al. [27] was relatively low (4.2\%). The $\mathrm{CHA}_{2} \mathrm{DS}_{2}$-VASc score was higher in patients undergoing anticoagulation than in those not undergoing anticoagulation in both studies. Similarly, the CCI scores were slightly lower in patients not undergoing anticoagulation in both studies [27].

Because of the similarity of our study with the FRAILAF study by Lefebvre et al. [28], we compared the data between the two studies. Both studies again included very old patients. In the study by Lefebvre et al. [28], a mean age of 87.4 years was observed in the group of patients not undergoing anticoagulation. Patients undergoing anticoagulation had a mean age of 85.3 years. The patient population in the study by Lefebvre et al. [28] was somewhat younger than in the present study, in which the average age was 90.6 years and patients treated with OACs tended to be younger than the patients who were not undergoing anticoagulation. Lefebvre et al. [28] reported that $70.0 \%$ of the patients were undergoing anticoagulation, and the corresponding proportion was $67.3 \%$ in the AVOPA study. Furthermore, it was shown that the proportion of patients undergoing anticoagulation increased with an increasing $\mathrm{CHADS}_{2}$ score and decreased with an increasing HAS-BLED score. This was also observed in our group comparison of patients taking and not taking OACs. Accordingly, patients taking OACs had a relatively higher $\mathrm{CHA}_{2} \mathrm{DS}_{2}$-VASc score and a lower HAS-BLED score [28].

\section{Limitations}

Regarding the limitations of the AVOPA study, it should be noted that this was a retrospective study. The individual parameters were determined by reviewing the patient charts. Thus, it was not possible to ask for specific criteria. However, the retrospective study design allowed a more objective evaluation of the data than a similarly designed prospective study. Furthermore, the present study was a single-center study that included data from the Geriatric Department of the Nuremberg Clinic. However, with an $n$ of 407 , the number of patients included in the study was sufficiently large. Because of the inclusion criterion of an age $>85$ years, the present study included a very specific patient population. Especially with regard to the older age and the numerous comorbidities in the study population, the results cannot be applied to the general population. However, it was our intention to investigate this specific, very old patient population to supplement the limited data available. Another limitation of the study is that no data were collected on the persistence of treatment with OACs after hospitalization; therefore, no statement about prognosis can be made. However, this was necessary to create a common starting and ending point for the study and to be able to make a statement about the choice of drug. As this was a retrospective study, data on when AF was diagnosed were not always available. However, patients were included only if they had AF diagnosed based on an existing electrocardiogram.

\section{Conclusions}

Because the international literature on the use of OACs in very old patients is limited, we chose the inclusion criterion of age $>85$ years to select exactly this age group of patients. Data from the AVOPA study show that $67.3 \%$ of the patients were undergoing anticoagulation. At the same time, the percentage of patients with DOACs increased, and the percentage of patients with phenprocoumon decreased during hospitalization. Apixaban was the most common DOAC. This shows the special importance of guidelines such as the OAC-FORTA 2016 for the best possible drug care of these very old patients.

Dose adjustment criteria are also an important issue in the very old patient group. In our study population, there was an alarming proportion of the patients who received an inappropriately low dose. There is potential for improvement with regard to dosage.

The AVOPA study could help to find a benchmark in the international comparison of the use of OACs in very old patients and the assessment of the quality of treatment with OACs at different hospitals. The proportion of very old patients with too low a dose is an important sub-area that should be examined more closely in further studies. In addition, there is a considerable proportion with a dose that is too high, this sub-area is also an important aspect for future studies. Furthermore, there is a particular complexity with regard to very old patients (age $>85$ years), especially with regard to the numerous comorbidities and the special effects of the frequently restricted kidney function on the DOAC, 
and therefore requires a precise future investigation to enable the best possible care for this group of patients.

Supplementary Information The online version contains supplementary material available at https://doi.org/10.1007/s40801-021-00263-6.

\section{Declarations}

Funding No sources of funding were used for the conduct of this study or the preparation of this article.

Conflict of interest Maximilian Hupfer has no conflicts of interest that are directly relevant to the content of this article. Markus Gosch received lecture fees from Pfizer, Bristol-Myers Squibb, Daiichi-Sankyo, and Bayer.

Ethics approval Approval was given by the institutional review board of the hospital.

\section{Consent to participate Not applicable.}

Consent for publication Not applicable.

Availability of data and material The data that support the findings of this study are available from the corresponding author upon reasonable request.

Author contributions HM and GM conceived of and designed the study. HM collected and organized the patient data from the patient files. HM and GM analyzed the data and contributed to the interpretation of the data. HM was a major contributor to writing the manuscript. All authors read and approved the final manuscript.

Open Access This article is licensed under a Creative Commons Attribution-NonCommercial 4.0 International License, which permits any non-commercial use, sharing, adaptation, distribution and reproduction in any medium or format, as long as you give appropriate credit to the original author(s) and the source, provide a link to the Creative Commons licence, and indicate if changes were made. The images or other third party material in this article are included in the article's Creative Commons licence, unless indicated otherwise in a credit line to the material. If material is not included in the article's Creative Commons licence and your intended use is not permitted by statutory regulation or exceeds the permitted use, you will need to obtain permission directly from the copyright holder. To view a copy of this licence, visit http://creativecommons.org/licenses/by-nc/4.0/.

\section{References}

1. Wilke T, Groth A, Mueller S, Matthias P, Frank V, Roland L, et al. Incidence and prevalence of atrial fibrillation: an analysis based on 8.3 million patients. EP Europace. 2013;15(4):486-93. https:// doi.org/10.1093/europace/eus333.

2. Lee K-N, Choi J-I, Boo KY, Kim DY, Kim YG, Oh S-K, et al. Effectiveness and safety of off-label dosing of non-vitamin $\mathrm{K}$ antagonist anticoagulant for atrial fibrillation in Asian patients. Sci Rep. 2020;10:1801. https://doi.org/10.1038/s41598-020-58665-5.

3. Zoni-Berisso M, Lercari F, Carazza T, Domenicucci S. Epidemiology of atrial fibrillation: European perspective. Clin Epidemiol. 2014;6:213-20. https://doi.org/10.2147/clep.s47385.
4. Chugh SS, Havmoeller R, Narayanan K, Singh D, Rienstra M, Benjamin EJ, et al. Worldwide epidemiology of atrial fibrillation: a Global Burden of Disease 2010 Study. Circulation. 2014;129:837-47. https://doi.org/10.1161/CIRCULATIONAHA. 113.005119

5. Colilla S, Crow A, Petkun W, Singer DE, Simon T, Liu X. Estimates of current and future incidence and prevalence of atrial fibrillation in the U.S. adult population. Am J Cardiol. 2013;112:1142-7. https://doi.org/10.1016/j.amjcard.2013.05.063.

6. Björck S, Palaszewski B, Friberg L, Bergfeldt L. Atrial fibrillation, stroke risk, and warfarin therapy revisited. Stroke. 2013;44(11):3103-8. https://doi.org/10.1161/strokeaha.113. 002329.

7. Chao TF, Liu CJ, Lin YJ, Chang SL, Lo LW, Hu YF, et al. Oral anticoagulation in very elderly patients with atrial fibrillation: a nationwide cohort study. Circulation. 2018;138:37-47. https://doi. org/10.1161/CIRCULATIONAHA.117.031658.

8. Granger CB, Alexander JH, McMurray JJV, Renato DL, Elaine $\mathrm{MH}$, Michael $\mathrm{H}$, et al. Apixaban versus warfarin in patients with atrial fibrillation. N Engl J Med. 2011;365(11):981-92. https:// doi.org/10.1056/nejmoa1107039.

9. Patel MR, Mahaffey KW, Garg J, Guohua P, Daniel ES, Werner $\mathrm{H}$, et al. Rivaroxaban versus warfarin in nonvalvular atrial fibrillation. N Engl J Med. 2011;365(10):883-91. https://doi.org/10. 1056/nejmoa1009638.

10. Connolly SJ, Ezekowitz MD, Yusuf S, Eikelboom J, Oldgren J, Parekh A, et al. Dabigatran versus warfarin in patients with atrial fibrillation. N Engl J Med. 2009;361(12):1139-51. https://doi.org/ 10.1056/nejmoa0905561.

11. Giugliano RP, Ruff CT, Braunwald E, Sabina AM, Stephen DW, Jonathan LH, et al. Edoxaban versus warfarin in patients with atrial fibrillation. N Engl J Med. 2013;369(22):2093-104. https:// doi.org/10.1056/nejmoa1310907.

12. Ruff CT, Giugliano RP, Braunwald E, Hoffman EB, Deenadayalu N, Ezekowitz MD, et al. Comparison of the efficacy and safety of new oral anticoagulants with warfarin in patients with atrial fibrillation: a meta-analysis of randomised trials. Lancet. 2014;383:955-62. https://doi.org/10.1016/s0140-6736(13) 62343-0.

13. Zulkifly H, Lip GYH, Lane DA. Epidemiology of atrial fibrillation. Int J Clin Pract. 2018;72(3):e13070. https://doi.org/10.1111/ ijcp. 13070.

14. Müssigbrodt A, Richter S, Hindricks G, Bollmann A. Vorhofflimmern bei Ausdauersportlern. Deutsche Zeitschrift für Sportmedizin. 2010;61:4-14.

15. Hart RG, Eikelboom JW, Brimble KS, McMurtry MS, Ingram AJ. Stroke prevention in atrial fibrillation patients with chronic kidney disease. Can J Cardiol. 2013;29:S71-8. https://doi.org/10.1016/j. cjca.2013.04.005.

16. Kirchhof P, Benussi S, Kotecha D, Ahlsson A, Atar D, Casadei B, ESC Scientific Document Group, et al. 2016 ESC guidelines for the management of atrial fibrillation developed in collaboration with EACTS. Eur Heart J. 2016;37:2893-962. https://doi.org/10. 1093/eurheartj/ehw210.

17. Steinberg BA, Blanco RG, Ollis D, Sunghee K, DaJuanicia NH, Peter RK, ORBIT-AF Steering Committee Investigators, et al. Outcomes registry for better informed treatment of atrial fibrillation II: rationale and design of the ORBIT-AF II registry. Am Heart J. 2014;168(2):160-7. https://doi.org/10.1016/j.ahj.2014. 04.005 .

18. Mahoney FI, Barthel DW. Functional evaluation: the Barthel Index. Md State Med J. 1965;14:61-5.

19. Granger CV, Dewis LS, Peters NC, Sherwood C, Barrett J. Stroke rehabilitation: analysis of repeated Barthel index measures. Arch Phys Med Rehabil. 1979;60:14-7. 
20. Shah S, Vanclay F, Cooper B. Improving the sensitivity of the Barthel Index for stroke rehabilitation. J Clin Epidemiol. 1989;42:703-9.

21. Gage BF, Waterman AD, Shannon W, Boechler M, Rich MW, Radford MJ. Validation of clinical classification schemes for predicting stroke results from the National Registry of atrial fibrillation. JAMA. 2001;285:2864-70. https://doi.org/10.1001/jama. 285.22.2864.

22. Lip GY, Nieuwlaat R, Pisters R, Lane DA, Crijns HJ. Refining clinical risk stratification for predicting stroke and thromboembolism in atrial fibrillation using a novel risk factor-based approach: the Euro Heart Survey on atrial fibrillation. Chest. 2010;137:26372. https://doi.org/10.1378/chest.09-1584.

23. Pisters R, Lane DA, Nieuwlaat R, de Vos CB, Crijns HJGM, Lip GYH. A novel user-friendly score (HAS-BLED) to assess 1-year risk of major bleeding in patients with atrial fibrillation: the Euro Heart Survey. Chest. 2010;138:1093-100. https://doi.org/10.1378/ chest.10-0134.

24. Charlson ME, Pompei P, Ales KL, MacKenzie CR. A new method of classifying prognostic comorbidity in longitudinal studies: development and validation. J Chronic Dis. 1987;40:373-83. https://doi.org/10.1016/0021-9681(87)90171-8.

25. Kirchhof P, Nabauer M, Gerth A, Limbourg T, Lewalter T, Goette A, AFNET Registry Investigators, et al. Impact of the type of centre on management of AF patients: surprising evidence for differences in antithrombotic therapy decisions. Thromb Haemost. 2011;105:1010-23. https://doi.org/10.1160/TH11-02-0070.

26. Barnes GD, Lucas E, Alexander GC, Goldberger ZD. National trends in ambulatory oral anticoagulant use. Am J Med. 2015;128:1300-1305.e2. https://doi.org/10.1016/j.amjmed.2015. 05.044 .

27. Ekerstad N, Karlsson T, Soderqvist S, Karlson BW. Hospitalized frail elderly patients - atrial fibrillation, anticoagulation and 12 months' outcomes. Clin Interv Aging. 2018;13:749-56. https:// doi.org/10.2147/CIA.S159373.

28. Lefebvre MC, St-Onge M, Glazer-Cavanagh M, Bell L, Kha Nguyen JN, Viet-Quoc Nguyen P, et al. The effect of bleeding risk and frailty status on anticoagulation patterns in octogenarians with atrial fibrillation: the FRAIL-AF study. Can J Cardiol. 2016;32:169-76. https://doi.org/10.1016/j.cjca.2015.05.012.

29. Wehling M, Collins R, Gil VM, Hanon O, Hardt R, Hoffmeister $\mathrm{M}$, et al. Appropriateness of oral anticoagulants for the longterm treatment of atrial fibrillation in older people: results of an evidence-based review and international consensus validation process (OAC-FORTA 2016). Drugs Aging. 2017;34:499-507. https://doi.org/10.1007/s40266-017-0466-6.

30. Wehling M, Burkhardt H, Kuhn-Thiel A, Pazan F, Throm C, Weiss C, Frohnhofen H. VALFORTA: a randomised trial to validate the FORTA (Fit fOR The Aged) classification. Age Ageing. 2016;45:262-7. https://doi.org/10.1093/ageing/afv200. 